\title{
Nuclear morphometry in indeterminate thyroid nodules
}

\author{
Michael A. Razavi ${ }^{1}$, Johnny Wong ${ }^{1}$, Mounika Akkera ${ }^{1}$, Mahmoud Shalaby $^{1}$, Hosam Shalaby ${ }^{1}$, \\ Andrew Sholl ${ }^{2}$, Antione Haddad ${ }^{1}$, Preeti Behl ${ }^{2}$, Emad Kandil ${ }^{1}$, Grace S. Lee ${ }^{1}$ \\ ${ }^{1}$ Division of Endocrine and Oncologic Surgery, Department of Surgery, ${ }^{2}$ Department of Pathology, Tulane University School of Medicine, New \\ Orleans, LA, USA \\ Contributions: (I) Conception and design: A Haddad, MA Razavi, A Sholl, GS Lee, E Kandil; (II) Administrative support: A Sholl, E Kandil, GS Lee; \\ (III) Provision of study materials or patients: A Sholl, E Kandil, A Haddad, GS Lee; (IV) Collection and assembly of data: MA Razavi, J Wong, M \\ Akkera, M Shalaby, H Shalaby, P Behl; (V) Data analysis and interpretation: All authors; (VI) Manuscript writing: All authors; (VII) Final approval of \\ manuscript: All authors. \\ Correspondence to: Grace S. Lee, MD. Department of Surgery, Tulane University School of Medicine, 1430 Tulane Avenue, SL-22, New Orleans, LA \\ 70112, USA. Email: glee3@tulane.edu.
}

\begin{abstract}
Backsround Up to $30 \%$ of thyroid nodules undergoing fine needle aspiration (FNA) yield an indeterminate result. Recent research efforts have suggested that nuclear morphometry and morphology may enhance the diagnostic accuracy of FNA as an objective adjunct. We applied nuclear morphometric analysis on a diverse cohort of patients to evaluate the association between nuclear morphometry and malignancy.

Methods: Forty-five randomly selected patients, who underwent thyroid surgery after an indeterminate FNA result (Bethesda III \& IV) between 2012-2015, were reviewed. One hundred representative nuclei per FNA of a thyroid nodule were analyzed using ImageJ. Seven validated morphometric parameters were collected: nuclear area, perimeter, circularity, aspect ratio, roundness, and maximum/minimum Feret's diameter. L/S ratio was subsequently calculated. All 8 nuclear parameters were reported as averages with standard errors of the mean (SEM). A Student's $t$-test was used to assess the association of nuclear parameters with final surgical pathology.
\end{abstract}

Results: The mean age of all patients was $56.31 \pm 15.39$ years, with female patients comprising $68.9 \%$ of the cohort. Twenty-two patients had malignant thyroid nodules. The mean perimeter of nuclei for the cohort was $18.48 \pm 0.45 \mu \mathrm{m}$, the mean area was $22.19 \pm 0.93 \mu \mathrm{m}$, and the mean maximum Feret's diameter was $6.67 \pm$ $0.13 \mu \mathrm{m}$. No significant differences in the 8 nuclear parameters were observed between the malignant and non-malignant groups.

Conclusions: In the population examined, our results suggest that nuclear morphometry is not yet a tool of reliable diagnostic value in accessing malignant and non-malignant thyroid nodules. Further investigation is necessary to identify objective parameters that will enhance diagnostic accuracy of indeterminate FNA cytology to minimize the number of diagnostic thyroid surgery.

Keywords: Fine needle aspiration (FNA); nuclear morphometry; indeterminate thyroid nodule

Submitted Oct 24, 2019. Accepted for publication Jan 17, 2020.

doi: $10.21037 /$ gs.2020.02.02

View this article at: http://dx.doi.org/10.21037/gs.2020.02.02

\section{Introduction}

Thyroid nodules are common findings in clinical practice with a reported prevalence of up to $65 \%$ in the general population (1). As these nodules can represent both benign and malignant pathology, further evaluation is required with a comprehensive ultrasound exam and fine needle aspiration (FNA), if indicated. FNA cytology guides the further management algorithm of thyroid nodules, including a diagnostic or therapeutic thyroid surgery.

FNA is performed by introducing a small caliber needle, generally $25-27 \mathrm{G}$, into the thyroid nodule to 


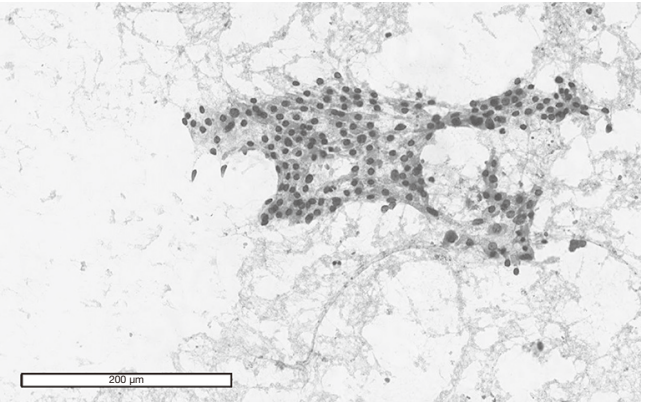

Figure 1 A representative FNA slide imaged at 20× magnification, bar $=200 \mu \mathrm{m}$. Papanicolaou (PAP) stained. FNA, fine needle aspiration.

aspirate parenchymal cells for microscopic examination (2). Pathologists assess the cytological characteristics, particularly the nuclear features associated with malignancy. The Bethesda System for Reporting Thyroid Cytopathology (TBSRTC) is the most widely utilized system for classifying thyroid cytopathology in the U.S. with six classification categories: (I) non-diagnostic or unsatisfactory, (II) benign, (III) atypia of undetermined significance, (IV) follicular neoplasm or suspicious for follicular neoplasm, (V) suspicious for malignancy, and (VI) malignant (3).

While the specificity and sensitivity of FNA to detect malignancy is up to $96.6 \%$ and $88.5 \%$, respectively, FNA alone is not adequate for diagnosing follicular and medullary carcinoma $(4,5)$. The rate of indeterminate thyroid nodules (Bethesda III \& IV) has been reported to be approximately $30 \%$, with significant institutional and regional variation (6). These thyroid nodules then further undergo an interval repeat FNA, expensive molecular testing, or a diagnostic surgery. As such, there is a risk of subjecting potentially benign pathology to molecular testing and surgery. Although thyroid surgery is a safe, frequently performed procedure with a very low complication rate, a central neck scar remains for the patient, adversely affecting quality of life and increasing the healthcare cost burden. A method to enhance the diagnostic accuracy of indeterminate FNA cytology to prevent further diagnostic thyroid surgery would be of great value.

The use of nuclear morphometry has been studied across many disciplines of surgical oncology, including breast and prostate $(7,8)$. Prior studies have investigated the diagnostic role of nuclear morphometry in an effort to predict thyroid cancer in certain populations (9). However, the use of nuclear morphometry to specifically evaluate indeterminate thyroid cytology is not well established. We examined the association between nuclear morphometry and malignancy in our cohort of indeterminate FNAs. We hypothesized that supplementing conventional FNA with nuclear morphometry in evaluating indeterminate thyroid nodules may be a useful tool in identifying malignancy in need of surgical intervention.

\section{Methods}

\section{Patient selection}

Forty-five patients, who underwent thyroid surgery after an indeterminate FNA (Bethesda III \& IV) between August 2012 to December 2015, were selected at random for this study. FNA slides from all patients were collected from the Pathology \& Cytology Office at the Medical Center. Patients were identified based on specimen number that was paired with their FNA procedure.

\section{Imaging}

Digital images of entire Papanicolaou (PAP) stained FNA slides were captured under $20 \times$ magnification using Aperio Digital Slide Scanner (Figure 1). Upon digitizing all FNA slides, the image files were exported and analyzed using ImageJ software.

\section{Image scaling \& analysis}

Slide images in TIFF files were opened in ImageJ for calibration. One hundred representative nuclei per FNA of a thyroid nodule were measured using ImageJ's analyze particles function for threshold set images. A group of nonoverlapping intact nuclei were selected for analysis with ImageJ's wand tool. The selected nuclei were analyzed using ImageJ's analyze particles function after thresholding, where the output yielded our measurements of interest in micrometers. The ImageJ output was then exported to Microsoft Excel to calculate the Length to Width ratio (L/S ratio) and other morphometric parameters. All data collectors were blinded to the final surgical pathology, when preforming nuclear measurement. A list of nuclear parameter definitions is in Figure 2.

\section{Statistical analysis}

The total population was described using means (standard deviation) for continuous variables and frequencies 


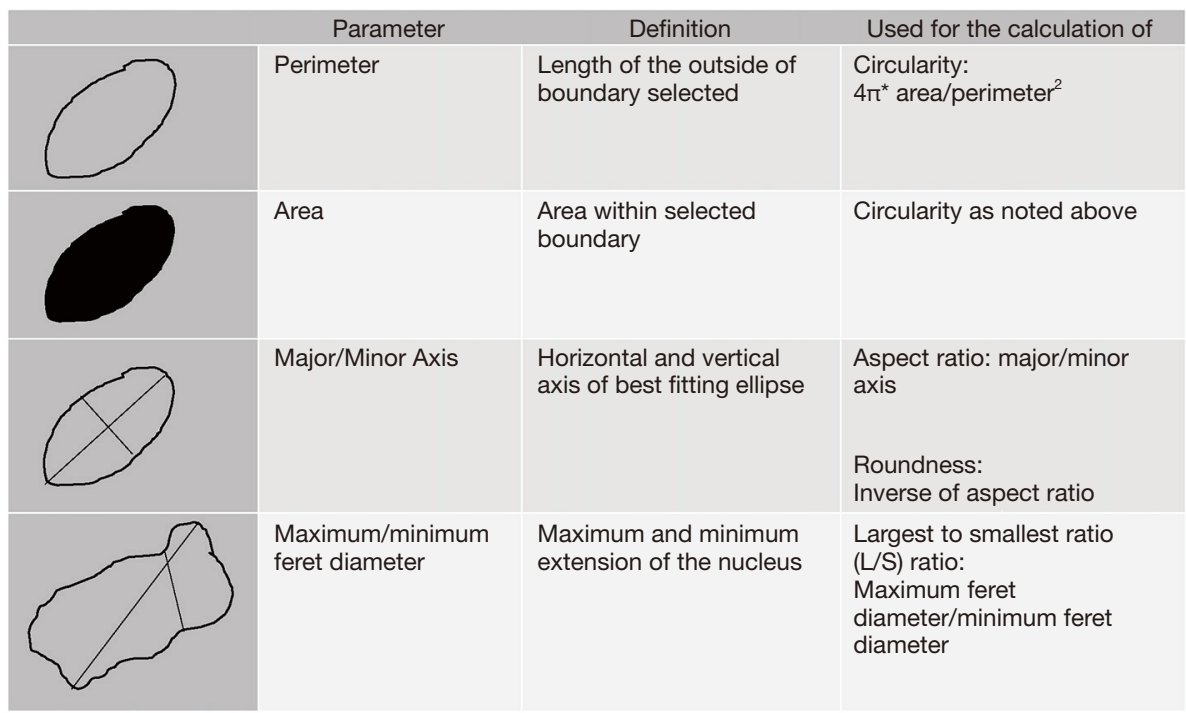

Figure 2 Description of nuclear morphometric parameters.

Table 1 Demographic and preoperative characteristics

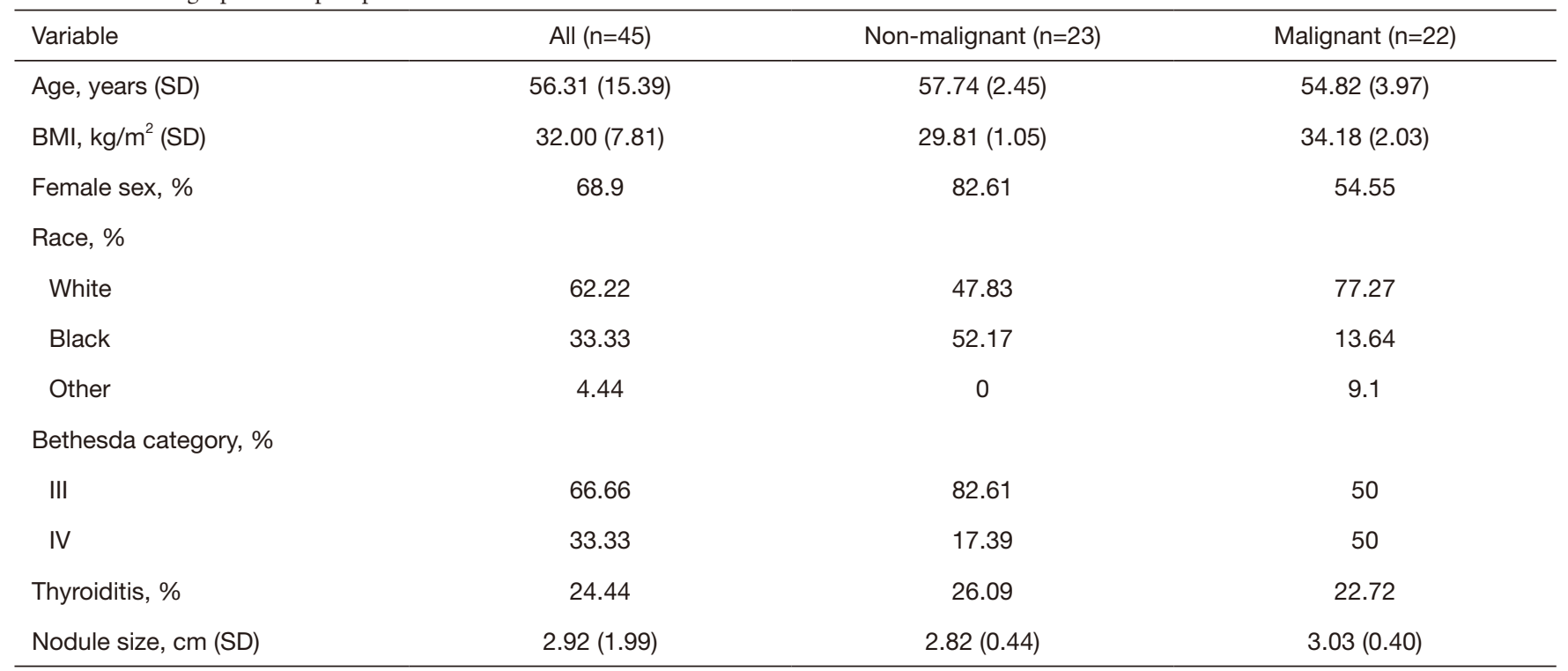

(percentage) for categorical variables. A Student's $t$-test was performed to assess the association of the individual nuclear parameters with malignancy per final surgical pathology (SAS v. 9.3, Cary, NC, USA). Statistical significance was defined by $\mathrm{P}$ value $<0.05$.

\section{Results}

The characteristics of the 45 patients included in this study are shown in Table 1. On average our cohort was middleaged and overweight with mean values of $56.31 \pm 15.39$ years in age and BMI of $32 \pm 7.81 \mathrm{~kg} / \mathrm{m}^{2}$, respectively. Female patients represented $68.90 \%$ and white patients comprised $62.22 \%$ of our study population. Twenty-two of our patients had malignant thyroid nodules and 23 of our patients had benign nodules per final surgical pathology. Our average nodule size for the entire cohort was $2.92 \pm 1.99 \mathrm{~cm}$ and $24.44 \%$ of our cohort had thyroiditis; $66.66 \%$ of our study 
Table 2 Mean nuclear morphometric measurements for all patients

\begin{tabular}{|c|c|c|c|c|}
\hline Parameter & All $(n=45)(\mu \mathrm{m} \pm$ SEM $)$ & Non-malignant $(n=23)(\mu \mathrm{m} \pm$ SEM) & Malignant $(n=22)(\mu \mathrm{m} \pm$ SEM) & $P$ value ${ }^{\dagger}$ \\
\hline Perimeter & $18.48 \pm 0.45$ & $18.70 \pm 0.69$ & $18.25 \pm 0.57$ & 0.62 \\
\hline Maximum Feret & $6.67 \pm 0.13$ & $6.76 \pm 0.20$ & $6.59 \pm 0.16$ & 0.52 \\
\hline Minimum Feret & $4.91 \pm 0.10$ & $4.96 \pm 0.16$ & $4.85 \pm 0.13$ & 0.58 \\
\hline Aspect ratio & $1.36 \pm 0.01$ & $1.36 \pm 0.01$ & $1.36 \pm 0.03$ & 0.92 \\
\hline Roundness & $0.77 \pm 0.01$ & $0.77 \pm 0.01$ & $0.77 \pm 0.01$ & 0.87 \\
\hline LS ratio & $1.38 \pm 0.01$ & $1.37 \pm 0.01$ & $1.38 \pm 0.02$ & 0.95 \\
\hline
\end{tabular}

${ }^{\dagger}, \mathrm{P}$ value for comparison between non-malignant and malignant cases.

Table 3 Mean nuclear morphometric measurements for patients with nodule $\leq 2 \mathrm{~cm}$ per preoperative ultrasound

\begin{tabular}{|c|c|c|c|c|}
\hline Parameter & All $(n=19)(\mu \mathrm{m} \pm$ SEM $)$ & Non-malignant $(n=11)(\mu \mathrm{m} \pm$ SEM) & Malignant $(n=8)(\mu \mathrm{m} \pm$ SEM $)$ & $P$ value ${ }^{\dagger}$ \\
\hline Perimeter & $18.16 \pm 0.44$ & $17.57 \pm 0.56$ & $18.96 \pm 0.63$ & 0.12 \\
\hline Maximum Feret & $6.60 \pm 0.14$ & $6.43 \pm 0.19$ & $6.84 \pm 0.17$ & 0.14 \\
\hline Minimum Feret & $4.84 \pm 0.12$ & $4.70 \pm 0.15$ & $5.03 \pm 0.20$ & 0.19 \\
\hline Aspect ratio & $1.36 \pm 0.03$ & $1.36 \pm 0.02$ & $1.37 \pm 0.07$ & 0.98 \\
\hline Round & $0.76 \pm 0.01$ & $0.76 \pm 0.01$ & $0.77 \pm 0.03$ & 0.84 \\
\hline L/S ratio & $1.38 \pm 0.02$ & $1.38 \pm 0.02$ & $1.39 \pm 0.05$ & 0.90 \\
\hline
\end{tabular}

${ }^{\dagger}, \mathrm{P}$ value for comparison between non-malignant and malignant cases.

population had nodules classified as Bethesda category III (Table 1).

Averages, with standard errors of the mean (SEM), for the eight nuclear parameters across the non-malignant and malignant patient groups are shown in Table 2. Examining the entire cohort showed a mean perimeter of $18.48 \pm 0.45 \mu \mathrm{m}$, a mean area of $22.19 \pm 0.93 \mu \mathrm{m}$, and a mean maximum Feret's diameter of $6.67 \pm 0.13 \mu \mathrm{m}$. Among the 22 cases with malignant pathology the mean nuclear perimeter was $18.25 \pm 0.57$, the mean area was $21.51 \pm 1.06$, and the mean maximum Feret's diameter was $6.59 \pm 0.16$. Values for these nuclear parameters in the non-malignant groups were similar. There were no significant differences between the malignant and non-malignant groups in any of the eight nuclear parameters measured. Subgroup analyses accounting for nodule size and thyroiditis yielded similar results (Tables 3,4).

\section{Discussion}

Thyroid nodules with indeterminate cytology pose challenges in further diagnostic workup. Forty-nine percent of our cohort underwent a diagnostic thyroid surgery for benign pathology, subjecting themselves to the possibility of surgical complications and financial burden in terms of medical payment and lost wages. Though recent advancements in thyroid cancer molecular genetics have led to the creation of molecular testing on cytology specimen, these tests may still be inconclusive and carry a large financial burden for patients (10).

We evaluated nuclear morphometry data as a potential low-cost diagnostic adjunct for discerning malignancy 
Table 4 Mean nuclear morphometric measurements for patient with thyroiditis

\begin{tabular}{|c|c|c|c|c|}
\hline Parameter & All $(n=11)(\mu \mathrm{m} \pm$ SEM) & Non-malignant $(n=6)(\mu \mathrm{m} \pm$ SEM $)$ & Malignant $(n=5)(\mu \mathrm{m} \pm$ SEM $)$ & $P$ value ${ }^{\dagger}$ \\
\hline Perimeter & $18.20 \pm 1.05$ & $19.06 \pm 1.68$ & $17.17 \pm 1.15$ & 0.40 \\
\hline Maximum Feret & $6.64 \pm 0.33$ & $6.95 \pm 0.54$ & $6.28 \pm 0.29$ & 0.34 \\
\hline Minimum Feret & $4.82 \pm 0.27$ & $5.10 \pm 0.43$ & $4.49 \pm 0.29$ & 0.28 \\
\hline Aspect ratio & $1.39 \pm 0.04$ & $1.36 \pm 0.03$ & $1.43 \pm 0.08$ & 0.46 \\
\hline Round & $0.76 \pm 0.02$ & $0.77 \pm 0.01$ & $0.74 \pm 0.03$ & 0.42 \\
\hline $\mathrm{L} / \mathrm{S}$ ratio & $1.41 \pm 0.03$ & $1.38 \pm 0.03$ & $1.43 \pm 0.06$ & 0.44 \\
\hline
\end{tabular}

${ }^{\dagger}, \mathrm{P}$ value for comparison between non-malignant and malignant cases.

status in indeterminate thyroid nodules. Our current results suggest that there is no significant difference in morphometric measurements between benign and malignant nodules from the indeterminate thyroid nodule cohort. The urgent need for a better diagnostic tool still remains for nodules in Bethesda categories III and IV.

In this study, nuclear morphometry information was not of significant diagnostic value; however, this is a discordant finding to other published studies. Prior studies have suggested that nuclear morphometry data are valuable adjuncts in determining the malignancy status of thyroid nodules. Parameters such as nuclear area, perimeter, L/S ratio, and minimum and maximum Feret's diameter were found to be significantly different between malignant and benign thyroid nodules $(9,11,12)$. Yashaswini et al. reported that malignant nodules had both larger areas and perimeters as compared to benign nodules, showing that morphometry provided preoperative diagnostic value (9). Furthermore, utilizing ultrasound images, Nugroho et al. noted that circularity and aspect ratio can be used to correctly classify the malignancy status of thyroid nodules (13).

The accuracy of FNA in large thyroid nodules remains controversial. Much of this uncertainty has been attributed to sampling error that is inherent when extracting cells during FNA, as diagnostic cells may be missed when sampling larger nodules. Prior surgical studies have reported that nodules less than $3 \mathrm{~cm}$ and those $\geq 3 \mathrm{~cm}$ carry false negative rates of $4.8 \%$ and $11.7 \%$, respectively (14). For the purposes of nuclear morphometry, the underlying premise is that FNA was performed accurately and diagnostic cells are available for analysis. To ensure that nodule size was not accounting for the lack of significance in our cohort, we performed a subgroup analysis on the 19 patients, who had nodules less than $2 \mathrm{~cm}$. No significant difference, however, was detected between benign and malignant cases across any of the 8 nuclear parameters (Table 3). In addition, we investigated whether underlying thyroiditis influenced morphometry results. Of the 11 patients who had thyroiditis, we again found no significant differences between the benign and malignant groups (Table 4).

There are many strengths to the current study. ImageJ is an easily accessible open-source image analysis platform available through the National Institutes of Health. By utilizing ImageJ's preset algorithms for obtaining nuclear measurements, our team was able to automate the data collection process and thus remove error that may be introduced with a manual method of data collection, while also obtaining data at a faster rate. All data collectors were blinded to the final surgical pathology, when slide scanning and performing nuclear measurement to remove any potential bias. Our study is not without limitations, however. Our sample size was small and there was a lack of diverse demographics in our malignant cases. Approximately $77 \%$ of our malignant cases were papillary thyroid cancers (PTC) with the remaining cases being follicular carcinomas. Overall, these malignancy cases were at an early stage with only one patient having lymph node metastasis. The homogeneity of this group, however, may have allowed for high internal validity within our study. We also acknowledge that digitizing our cytology slides at a low power (20x) magnification for larger field capturing may have posed a limitation in the degree of image resolution necessary to reflect adequate micro-scale morphometry measurements. In order to reduce selection bias of nuclei, 
we aimed to maximize the number of nuclei represented per FNA slide for analysis, as to not miss diagnostic cells (12).

Accurately identifying malignancy among indeterminate thyroid nodules would significantly minimize patient burden associated with diagnostic surgery, adverse effects on quality of life, and costs associated with molecular testing. A larger scale, population-based study utilizing more patient samples and nuclear imaging at higher magnification may potentially validate the utility of nuclear morphometry in thyroid nodule diagnosis. With increasing automation of image analysis and utilization of artificial intelligence technology in healthcare, identification of a validated, reliable, and cost-effective tool for interpreting indeterminate thyroid nodule cytopathology will greatly reduce patient morbidity and better utilize healthcare resources.

\section{Conclusions}

In the population examined, our results suggest that nuclear morphometry is not yet a tool of reliable diagnostic value in accessing malignant and non-malignant thyroid nodules. Further investigation is necessary to identify objective parameters that will enhance diagnostic accuracy of indeterminate FNA cytology to minimize the number of diagnostic thyroid surgery.

\section{Acknowledgments}

The authors would like to acknowledge Troy P. Taliancich for his technical assistance during this project.

Funding: None.

\section{Footnote}

Conflicts of Interest: All authors have completed the ICMJE uniform disclosure form (available at http://dx.doi. org/10.21037/gs.2020.02.02). EK serves as an unpaid Editor-in-Chief of Gland Surgery from Aug 2015 to Jul 2020. The other authors have no conflicts of interest to declare.

Ethical Statement: The authors are accountable for all aspects of the work in ensuring that questions related to the accuracy or integrity of any part of the work are appropriately investigated and resolved. Tulane University Medical Center Institutional Review Board approval was obtained for the completion of this study (IRB\# 2017-650-
TUHSC). Informed consent was not required as per IRB review.

Open Access Statement: This is an Open Access article distributed in accordance with the Creative Commons Attribution-NonCommercial-NoDerivs 4.0 International License (CC BY-NC-ND 4.0), which permits the noncommercial replication and distribution of the article with the strict proviso that no changes or edits are made and the original work is properly cited (including links to both the formal publication through the relevant DOI and the license). See: https://creativecommons.org/licenses/by-nc-nd/4.0/.

\section{References}

1. Dean DS, Gharib H. Epidemiology of thyroid nodules. Best Pract Res Clin Endocrinol Metab 2008;22:901-11.

2. Macedo AA, Pessotti HC, Almansa LF, et al. Morphometric information to reduce the semantic gap in the characterization of microscopic images of thyroid nodules. Comput Methods Programs Biomed 2016;130:162-74.

3. Mufti ST, Molah R. The bethesda system for reporting thyroid cytopathology: a five-year retrospective review of one center experience. Int J Health Sci (Qassim) 2012;6:159-73.

4. Thewjitcharoen Y, Butadej S, Nakasatien S, et al. Incidence and malignancy rates classified by The Bethesda System for Reporting Thyroid Cytopathology (TBSRTC) - An 8-year tertiary center experience in Thailand. J Clin Transl Endocrinol 2018;16:100175.

5. American Thyroid Association (ATA) Guidelines Taskforce on Thyroid Nodules and Differentiated Thyroid Cancer, Cooper DS, Doherty GM, et al. Revised American Thyroid Association management guidelines for patients with thyroid nodules and differentiated thyroid cancer. Thyroid 2009;19:1167-214.

6. Malheiros DC, Canberk S, Poller DN, et al. Thyroid FNAC: Causes of false-positive results. Cytopathology 2018;29:407-17.

7. Lim CN, Ho BC, Bay BH, et al. Nuclear morphometry in columnar cell lesions of the breast: is it useful? J Clin Pathol 2006;59:1283-6.

8. Hovens MC, Lo K, Kerger M, et al. 3D modelling of radical prostatectomy specimens: Developing a method to quantify tumor morphometry for prostate cancer risk prediction. Pathol Res Pract 2017;213:1523-9. 
9. Yashaswini R, Suresh TN, Sagayaraj A. Cytological Evaluation of Thyroid Lesions by Nuclear Morphology and Nuclear Morphometry. J Cytol 2017;34:197-202.

10. Ferris RL, Baloch Z, Bernet V, et al. American Thyroid Association Statement on Surgical Application of Molecular Profiling for Thyroid Nodules: Current Impact on Perioperative Decision Making. Thyroid 2015;25:760-8.

11. Karslioğlu Y, Celasun B, Günhan O. Contribution of morphometry in the differential diagnosis of fine-needle thyroid aspirates. Cytometry B Clin Cytom 2005;65:22-8.

12. Aiad H, Abdou A, Bashandy M, et al. Computerized

Cite this article as: Razavi MA, Wong J, Akkera M, Shalaby M, Shalaby H, Sholl A, Haddad A, Behl P, Kandil E, Lee GS. Nuclear morphometry in indeterminate thyroid nodules. Gland Surg 2020;9(2):238-244. doi: 10.21037/gs.2020.02.02 nuclear morphometry in the diagnosis of thyroid lesions with predominant follicular pattern.

Ecancermedicalscience 2009;3:146.

13. Nugroho HA, Frannita EL, Ardiyanto I, et al. Computer aided diagnosis for thyroid cancer system based on internal and external characteristics. J King Saud Univ Comput Inform Sci 2019. doi: 10.1016/ j.jksuci.2019.01.007.

14. Giles WH, Maclellan RA, Gawande AA, et al. False negative cytology in large thyroid nodules. Ann Surg Oncol 2015;22:152-7. 